\title{
Optimal motion planning for overhead cranes
}

\author{
Zhou Wu, Xiaohua Xia \\ Department of Electrical Electronic Computer Engineering \\ University of Pretoria, Pretoria, SA \\ Email: wuzhsky@gmail.com*,xxia@up.ac.za
}

July 9,2014

\begin{abstract}
Overhead cranes are widely used in industrial applications for material displacing. Many linear or nonlinear control schemes have been proposed for overhead cranes and implemented on electronic systems, but energy efficiency of transportation has seldom been considered in motion planning. This paper aims at finding an optimal solution of motion planning in terms of energy efficiency for overhead cranes. Using the optimal control method an optimal trajectory is obtained with less energy consumption than the compared trajectories and is also satisfying physical and practical constraints such as swing, acceleration, and jerk. Besides the energy optimal model, we also propose two other models to optimize time efficiency and safety during transportation. The results obtained have been compared with some existing motion trajectories, and have been shown to be superior to these benchmarks in terms of energy efficiency, time efficiency and safety respectively.
\end{abstract}

Keywords: Overhead crane; Optimal control; Motion planning; Energy efficiency

\section{Introduction}

In some industrial fields, such as sea ports, manufacturing plants, factories and construction sites, overhead cranes are indispensable for handling materials in the 
process of transportation and production $[1,2,3]$. Due to high payload capacity, good operational flexibility and efficient energy consumption, overhead cranes are playing significant roles in many industrial applications. Regardless of the type of overhead crane, overhead cranes always have a similar fundamental structure that can be described as a trolley-pendulum system. The overhead crane is a typical nonlinear underactuated mechanical system. The system, which consists of a trolley, a supporting frame and a rope connecting the trolley with the payload, has fewer independent control inputs than degrees of freedom. Compared with fully actuated systems, control of underactuated systems is much challenging. Therefore, underactuated overhead cranes have attracted much interest from researchers in areas of mechanics and control.

One complete crane motion process is composed of three phases: payload hoisting, horizontal transportation, and payload lowering, among which the control of horizontal transportation is the most difficult problem. These three phases can be integrated in one maneuver with overlap $[4,5,6,7]$ or be decomposed sequentially without overlap $[8,9]$. Phase overlap means that the payload can be horizontally transported when it is hoisted and that the payload can be lowered during the horizontal transportation. In this paper, phase overlap is not considered as the scope is specifically modeling motion planning problems confronted in the most complicated horizontal phase. Time efficiency and safety of the horizontal transportation are two main objectives of motion planning that are usually considered in previous work $[10,9]$. For time efficiency, the transportation time is preferred to be as short as possible while other safety and mechanical constraints must be satisfied. For safety, the requirement is to suppress or eliminate payload swing for some fragile or harmful payloads. To meet the safety requirement, both transient and residual swing must be controlled in a small tolerance range.

Many control strategies have been proposed to improve their performance in terms of time efficiency and transportation safety of overhead cranes. Due to the nonlinear nature of the crane system [11], nonlinear control methods, such as sliding-mode control [12], flatness-based inverse methods[4, 5, 7] and energy-based control scheme [13], have been applied to control overhead cranes. For simplicity, the original complicated crane systems are usually linearized for using linear control methods. Under the assumption of small payload swing, the nonlinear crane model can be linearized around its equilibrium points, and then linear control approaches can be used on the simplified linear system. Many linear control methods have been applied to overhead cranes, including feedback control $[14,15,16]$, input shaping (feedforward control) [17, 18, 19], optimal control [20, 21, 22]. Feedback control methods utilize measurements and estimates of the system states to reduce the payload's swing, while feedforward approaches alter the actuator commands so that system oscillations are reduced. Optimal control methods look for opti- 
mal solutions to minimize (or maximize) the defined objectives of crane problems subject to certain problem-based constraints.

In some control strategies, motion planning is a preliminary step to generate operation commands (profiles of acceleration or velocity) before online control of overhead cranes [23, 24]. Here motion planning means the trajectory design of trolley velocity and acceleration, which are regarded as control inputs (or states) of the crane system with velocity-driven or force-driven trolley. Unlike the inverse methods to control the stability of tracking the reference of outputs $[4,5]$, optimal control is a useful method to design the profile of control inputs with certain optimal performance index, such as time efficiency and swing angle. Optimal design of the transportation trajectory not only improves the control performance in terms of efficiency or safety, but also releases some pressure on control for the sequential strategy. Researchers have focused their efforts on motion planning of overhead cranes. The most difficult task in motion planning is to analyze the kinematic coupling behavior between the underactuated payload's swing and the actuated trolley's motion. In [20,21], optimal control theory was effective to generate optimal transportation trajectories that could minimize steady-state error or transportation time. A motion planning-based adaptive control strategy [25] was proposed to make the trolley track the planned S-shaped trajectory, where an online updating mechanism was employed to enhance the control stability under different working conditions. In [8], an anti-swing motion planning scheme with swing suppression was designed based on the anti-swing control law and typical crane operations in practice. In [9], three analytical trajectories were designed to address the coupling behavior and consider practical constraints such as swing amplitude, residual swing, and physical constraints.

Among existing literature on motion planning, some trajectories are designed according to the empirical experiences; some trajectories are designed for satisfying practical constraints; and the other trajectories are designed in consideration of time efficiency of motion planning. In [20], time optimal control theory has been evaluated on the bang-bang control system of cranes, but their time-optimal solution has not considered the continuous system and the constraint of swing amplitude. In [21, 22], optimal trajectories have been designed for continuous systems of cranes subject to the swing constraint. It may be noted that swing-optimal control is seldom considered to minimize the swing angle separately for safety in transportation. Another issue that has been neglected in the crane control is energy efficiency. Nowadays, the energy issue turns out to be significantly urgent as power demand keeps increasing when a large number of machines, such as belt conveyors and cranes, have been equipped in industry [26]. In the crane control, the profile of power, as well as the total energy consumption, is closely related to the transportation trajectory, so energy efficiency may be optimized by choosing 
a proper motion trajectory. These two issues will be analyzed and solved in our proposed strategies for overhead cranes.

In this paper, two objectives, safety and energy efficiency, are studied under the optimal control theory. First, swing-optimal model is proposed to minimize the swing amplitude and residual oscillation, in order to provide the highest degree of safety during horizontal transportation. Second, energy-optimal model is proposed to minimize the energy consumption in the transportation period. The power profile obtained is smoothed, and its peak is reduced as well. For the purpose of better comparison, we will revisit the time-optimal motion planning problem, but we include more practical constraints, such as maximum swing amplitude, and maximum jerk of motion. These three proposed motion planning strategies have covered the demanding requirements of crane control systems, and results obtained in the simulation section indicate their superiority in terms of time efficiency, safety and energy efficiency.

This paper is organized as follows. Section 2 describes the dynamic model of the overhead crane system. Then literature review on motion planning of overhead cranes is given in Section 3. In Section 4, the discrete-time dynamic equation is deduced and three optimal strategies in terms of the safety, energy efficiency and time efficiency are proposed. The comparison study is performed in Section 5. The last section concludes this paper.

\section{Description of the Overhead Crane System}

An overhead crane can be sketched as shown in Figure 1, where the trolley moves on the horizontal bridge and the payload is connected with a constant-length rope. $x(t), \theta(t)$ and $F(t)$ denote the trolley's position, the payload's swing angle and overall force on the trolley respectively. In this paper, stiffness and mass of the rope, as well as air resistance, are neglected and the load is considered as a point mass. Moreover, as this study only focuses on the open-loop optimal planning of horizontal transportation, hoisting and the effects of wind disturbance are not considered. Then the overhead crane system with constant rope length can be described as follows:

$$
\begin{gathered}
(M+m) \ddot{x}+m l \cos \theta \ddot{\theta}-m l \sin \theta \dot{\theta}^{2}=F, \\
m l^{2} \ddot{\theta}+m l \cos \theta \ddot{x}+m g l \sin \theta=0,
\end{gathered}
$$

where $M$ and $m$ denote masses of the trolley and the payload, respectively. $l$ is the length of the rope; $g$ the gravitational acceleration. The overall force $F$ is composed of the actuating force $F_{a}$ and the friction $F_{r}$ as

$$
F=F_{a}-F_{r},
$$




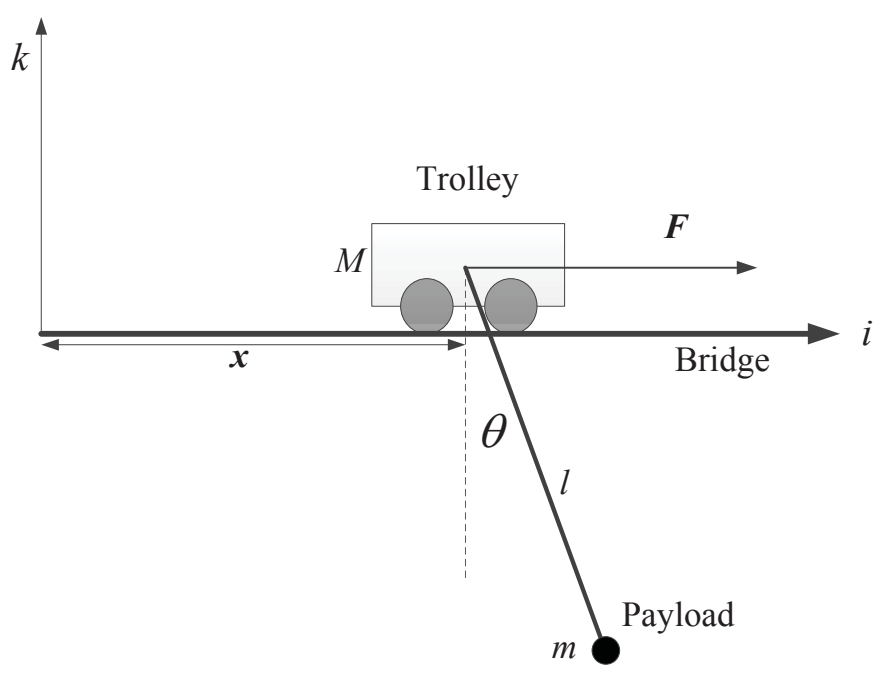

Figure 1: Two-dimensional overhead crane system

Motivated by the friction models in [27, 28], this paper employs the same nonlinear friction model as

$$
F_{r}=f_{r 0} \tanh \dot{x} / \xi-k_{r}|\dot{x}| \dot{x}
$$

where $f_{r 0}, k_{r} \in \mathbb{R}$ are friction-related parameters and $\xi \in \mathbb{R}$ is a static friction coefficient, which can be obtained from off-line experimental analysis. Note that the small friction caused by the payload's swing is neglected in the above model.

The payload-swing dynamics Eq. (2) defines the kinematic relationship between the trolley acceleration and the load swing. For this reason, the swing dynamics can be considered as a kinematic equation for motion planning and can be rewritten as

$$
l \ddot{\theta}+\cos \theta \ddot{x}+g \sin \theta=0 .
$$

When the swing angle is small enough $\left(\theta<5^{\circ}\right)$, the kinematic equation can be linearized with the approximations of $\cos \theta \simeq 1$ and $\sin \theta \simeq \theta$. The approximated linear kinematics can be obtained as

$$
l \ddot{\theta}+\ddot{x}+g \theta=0 .
$$

In the evaluated planning period $T$, a successful trajectory must ensure the crane to arrive at the destination. For a successful trajectory planning, several constraints must be satisfied according to practical constraints. 
(1): The trolley reaches the desired location $p_{d}$ at the end of the period. The final states of the payload must be static with no swing so that it can be lowered immediately as

$$
x(T)=p_{d}, \dot{x}(T)=0, \theta(T)=0, \dot{\theta}(T)=0 .
$$

(2): During the horizontal transportation, velocity and acceleration of the trolley must be limited in certain ranges as

$$
\left\{\begin{array}{l}
0 \leq \dot{x}(t) \leq v_{m} \\
|\ddot{x}(t)| \leq a_{m}
\end{array}, t \in(0, T]\right.
$$

where $v_{m}$ and $a_{m}$ are the permitted maximum of velocity and acceleration, respectively.

(3): The payload swing during the transportation must be limited within a safe range as

$$
|\theta(t)| \leq \theta_{m}, t \in(0, T]
$$

where $\theta_{m}$ is the permitted maximum of swing amplitude.

(4): The jerk (defined as the time derivative of acceleration $j(t)=\dddot{x}(t)$ ) must be limited to a reasonable range to satisfy the mechanical constraint and to prolong the motor's lifetime.

$$
|j(t)| \leq j_{m}, t \in(0, T]
$$

where $j_{m}$ is the permitted maximal jerk in the horizontal transportation.

\section{$3 \quad$ Literature Review}

Different models, as well as control methods of overhead cranes, have been proposed in the past decades. These have been well reviewed in [29]. As the scope of this paper focuses on the motion planning methods, five trajectory references mentioned in $[9,25,8]$ are analyzed in this section. For the same specific overhead system, we have plotted these five trajectories in Figure 2. For each trajectory, the profiles of velocity, acceleration and swing angle are shown in the figure.

\section{$3.1 \quad$ Trajectory 1}

Lee [8] proposed a smooth velocity profile for a smooth low-jerk motion. Lee's trajectory has been named Trajectory 1 as shown in Figure 2. When the trolley reaches the destination, the velocity and acceleration become 0 . Because the swing suppression has not been considered in Trajectory 1, the residual swing is unacceptably large. 

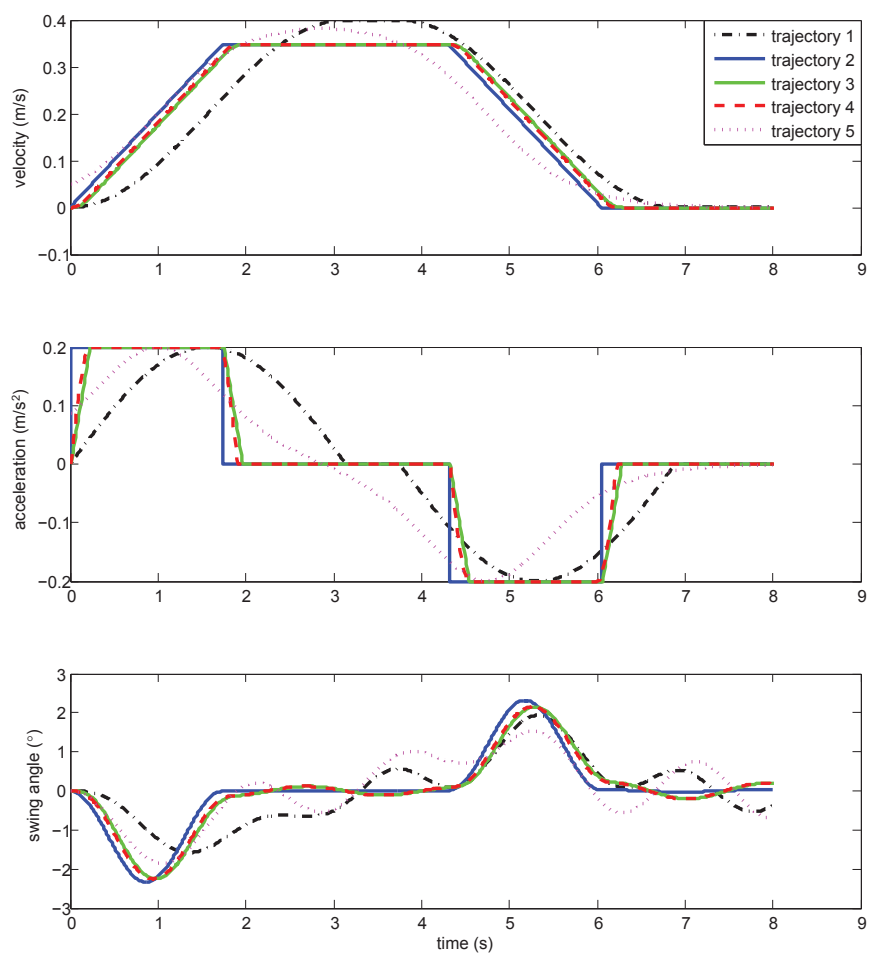

Figure 2: Trajectory references in terms of velocity, acceleration and swing angle

\subsection{Trajectory 2}

In $[30,9]$, a symmetric three-segment trajectory is proposed to eliminate the residual swing. From Figure 2, it can be noticed that Trajectory 2 has shorter arrival time than the other four trajectories. It has zero residual swing, and the jerk at the time $t_{2 a}, t_{2 a}+t_{2 c}$ and $2 t_{2 a}+t_{2 c}$ is infinite, which implies a discontinuous control signal and hence potential damage to the actuating motor.

\subsection{Trajectory 3}

In Trajectory 3 [9], some linear ramp functions at the transition stage are inserted into the three-segment trajectory to ensure smooth motion. It can be noticed from Figure 2 that the residual swing of Trajectory 3 is zero when the trolley stops. Unlike Trajectory 2 with infinite jerk, Trajectory 3 has jerk not larger than a predefined value during transportation. 


\subsection{Trajectory 4}

Like in Trajectory 3, ramp functions in Trajectory 4 [9] have also been inserted into the transient stages. The main difference is that the ramp functions are not linear but S-shaped functions. For Trajectory 4, the profiles of velocity, acceleration and swing angle are given in Figure 2. They are almost the same as the profiles of Trajectory 3 due to similar structures.

\subsection{Trajectory 5}

In [25], another S-curve trajectory is used as the reference for nonlinear tracking. Trajectory 5 satisfies the physical constraints of velocity and acceleration. The obtained trajectory always has the positive velocity bounded to the permitted maximum of velocity; its acceleration is also bounded to the permitted maximum of acceleration. It can be noticed from Figure 2 that the residual swing exists in Trajectory 5, and that the arriving time is longest among these referred trajectories. Another observation is that the initial velocity is not zero in Trajectory 5 , so the acceleration profile of Trajectory 5 cannot be used in open-loop controllers for the static cranes. Only closed-loop controllers (such as PID or fuzzy controllers) can use this profile of acceleration (or velocity) as the reference for tracking. As this paper only considers motion planning when the initial velocity of crane is zero, Trajectory 5 is not included in the simulation section.

\section{Optimal control models}

The five trajectories mentioned above have analytical formulations and convenient setting of parameters, which are easily applied to some real-time implementations. Although some of them have the advantage of zero residual swing, their swing amplitudes have not been optimized. Among these five trajectories, Trajectory 2 has the shortest transportation time, which is still not the minimal one. Another important issue is the fact that the energy consumption has not been considered yet in these trajectories. Therefore, it is necessary and meaningful to propose such trajectories that can minimize the transportation time, swing angle and energy consumption. In this section, three optimal control strategies are proposed to provide optimal solutions in terms of transportation safety, energy and time efficiency. Each proposed model is a discrete model with the input of acceleration. Therefore, the continuous system is first discretized by a sampling period $t_{0}$. Over the evaluated planning period $T=N \cdot t_{0}$, the discrete system of the overhead crane can be formulated as Eq. (32) and Eq. (33). 


$$
\begin{gathered}
(M+m) \Delta^{2} x(n)+m l \cos \theta(n) \Delta^{2} \theta(n)-m l \sin \theta(n) \Delta \theta(n)^{2}=F(n), \\
l \Delta^{2} \theta(n)+\Delta^{2} x(n)+g \theta(n)=0,
\end{gathered}
$$

where $n=1, \ldots, N ; x(n), \theta(n)$ and $F(n)$ represent the displacement, swing angle and overall force at the $n$th sample respectively. $\Delta$ and $\Delta^{2}$ represent the first and second-order difference as

$$
\left\{\begin{array}{l}
\Delta q(n)=(q(n+1)-q(n)) / t_{0} \\
\Delta^{2} q(n)=(\Delta q(n+1)-\Delta q(n)) / t_{0}
\end{array}, q(n)=[x(n), \theta(n)]^{T} .\right.
$$

In the description of proposed models, we denote the input vector (acceleration) as $\boldsymbol{a}\left(a(n)=\Delta^{2} x(n)\right)$, and denote the state vector of velocity as $\boldsymbol{v}(v(n)=\Delta x(n))$. When the initial state of the crane is static with zero swing, the velocity $\boldsymbol{v}$, the displacement $\boldsymbol{x}$ and the swing angle $\boldsymbol{\theta}$ can be expressed by the acceleration $\boldsymbol{a}$ as

$$
\left\{\begin{array}{l}
\boldsymbol{a}=[\ddot{x}(1), \ldots, \ddot{x}(N)]^{T} \\
\boldsymbol{v}=A_{v} \boldsymbol{a} t_{0} \\
\boldsymbol{x}=A_{x} \boldsymbol{a} t_{0}^{2} \\
\boldsymbol{\theta}=A_{\theta} \boldsymbol{a} t_{0}^{2}
\end{array},\right.
$$

where

$$
\begin{aligned}
& A_{v}=\left[\begin{array}{ccccc}
1 & 0 & 0 & \ldots & 0 \\
1 & 1 & 0 & \ldots & 0 \\
1 & 1 & 1 & \ldots & 0 \\
\vdots & \vdots & \vdots & \vdots & \vdots \\
1 & 1 & 1 & \ldots & 1
\end{array}\right] \\
& A_{x}=\left[\begin{array}{ccccc}
0.5 & 0 & 0 & \ldots & 0 \\
1.5 & 0.5 & 0 & \ldots & 0 \\
2.5 & 1.5 & 0.5 & \ldots & 0 \\
\vdots & \vdots & \vdots & \vdots & \vdots \\
N-0.5 & N-1.5 & N-2.5 & \ldots & 0.5
\end{array}\right] \text {, } \\
& A_{\theta}=-\frac{1}{l} B^{-1} \\
& =-\frac{1}{l}\left[\begin{array}{cccccc}
1+\frac{g t_{0}}{l} & 0 & \ldots & 0 & 0 & 0 \\
-2 & 1+\frac{g t_{0}}{l} & \ldots & 0 & 0 & 0 \\
1 & -2 & \ddots & 0 & 0 & 0 \\
0 & \ddots & \ddots & \ddots & \ddots & \vdots \\
0 & \ldots & \ddots & 1 & -2 & 1+\frac{g t_{0}}{l}
\end{array}\right]^{-1}
\end{aligned}
$$


Note that the matrix $B$ is non-singular. Eq. (14) is deduced according to Newton's second law of motion. Based on the discrete system, three optimal control models are then introduced as follows.

\section{(1) Swing-optimal model}

The objective function of swing-optimal model is about safety over the horizonal transportation. Safety of an overhead crane can be reflected by two metrics, i.e. the maximal swing angle and the residual swing. In the evaluated planning period $T$, the objective function will integrate both two metrics as

$$
J_{1}(\boldsymbol{a})=\alpha \max _{n \in\{1, \ldots, N\}} \theta(n)+(1-\alpha) \sum_{n=N-N_{r}+1}^{N} \frac{\theta(n)}{N_{r}}
$$

where $N_{r}$ is the number of samples considered in the residual swing. The first component of the right-hand side is the maximal swing angle, and the second component of the right-hand side is the residual swing. The coefficient $\alpha$ is used to integrate these two metrics. $\alpha$ is set to 0.5 in this paper. The residual swing is defined as the average swing angle during the final $N_{r} \cdot t_{0}$ period.

By substituting $\theta(n)$ with acceleration $a(n)$ using Eq. (14), the swing-optimal model Eq. (18) can be expressed by the acceleration. In this model, the input vector $\boldsymbol{a}$ is bounded in the range $\left[-a_{m}, a_{m}\right]$ as

$$
a(n) \in\left[-a_{m}, a_{m}\right], n=1, \ldots, N .
$$

The constraints of this model include the equality constraints of final displacement, velocity and swing angle, and also include the inequality constraints of velocity, swing angle and jerk. The set of constraints $\mathcal{C}(N)$ is formulated as

$$
\mathcal{C}(N): \begin{cases}-a_{m} \leq a(n) \leq a_{m}, & n=1, \ldots, N \\ x(N)=x_{p} & \\ v(N)=0 & \\ \theta(N)=0 & \\ \theta(N-1)=0 & n=1, \ldots, N \\ 0 \leq v(n) \leq k_{v}, & n=1, \ldots, N \\ |\theta(n)| \leq \theta_{\max }, & \\ |a(n+1)-a(n)| \leq j_{m}, & n=1, \ldots, N-1 \\ |a(1)| \leq j_{m} & \\ |a(N)| \leq j_{m} & \end{cases}
$$

To minimize the objective function Eq. (18) under the constraints, the solution is expected with the highest degree of safety.

(2) Energy-optimal model 
The objective function of energy-optimal model is energy consumption over the horizontal transportation. Within the planning period $T$, energy consumption can be calculated as

$$
E=\int_{0}^{T} P d t=\int_{0}^{T} F_{a} \dot{x} d t
$$

where $F_{a}$ is the output force of the actuating motor, $P$ is the power of the actuating motor, and $E$ is the energy consumption of the motor. For the discrete system, the proposed energy-optimal model can be formulated as

$$
J_{2}(\boldsymbol{a})=\sum_{n=1}^{N} F_{a}(n) v(n)=\sum_{n=1}^{N}\left[F(n)+F_{r}(n)\right] v(n)
$$

where the overall force $F(n)$ is computed as Eq. (11), and the friction $F_{r}(n)$ can be formulated similarly with Eq. (4) as

$$
F_{r}(n)=f_{r 0}(\tanh v(n) / \xi)-k_{r}|v(n)| v(n) .
$$

By substituting $v(n)$ and $\theta(n)$ with $a(n)$ using Eq. (14), the energy consumption can be expressed by the acceleration $\boldsymbol{a}$. The input vector is also bounded in $\left[-a_{m}, a_{m}\right]$. The energy optimal control model has the same constraints $\mathcal{C}(N)$ as the swing optimal control model. For the energy-optimal model, we aim to minimize the energy consumption in Eq. (22) under the practical constraints.

\section{(3) Time-optimal model}

The objective function of time-optimal model is equivalent with the transportation time. If the transportation time is denoted as $t_{f}=N_{f} \cdot t_{0}$, then the time-optimal model can be formulated as

$$
J_{3}(\boldsymbol{a})=N_{f}
$$

It is difficult to express $N_{f}$ by the acceleration $\boldsymbol{a}$. We can only determine $N_{f}$ by checking the feasibility of a specific acceleration profile. $\mathcal{C}(i)$ denotes constraints that must be satisfied when the transportation can be completed at $i \cdot t_{0} \cdot \mathcal{C}(i)$ is formulated as 


$$
\mathcal{C}(i): \begin{cases}-a_{m} \leq a(n) \leq a_{m}, & n=1, \ldots, i \\ x(i)=x_{p} & \\ v(i)=0 & \\ \theta(i)=0 & \\ \theta(i-1)=0 & n=1, \ldots, i \\ 0 \leq v(n) \leq k_{v}, & n=1, \ldots, i \\ |\theta(n)| \leq \theta_{\max }, & n=1, \ldots, i-1 \\ |a(n+1)-a(n)| \leq j_{m}, & \\ |a(1)| \leq j_{m} & \\ |a(i)| \leq j_{m} & \end{cases}
$$

If the profile satisfies the constraints $\mathcal{C}(i)$, the transportation time is not longer than $i \cdot t_{0} . N_{f}$ can be determined in a backward way (shown in Algorithm 1) or a bisection way (shown in Algorithm 2) to repeatedly check feasibility. If the transportation time is close enough to the planning period $T$, the backward algorithm can find $N_{f}$ effectively. Otherwise, the bisection algorithm is a better choice than the backward algorithm. Although $N_{f}$ can be known for any profile of acceleration, it is still difficult to find an efficient direction of decreasing $N_{f}$. In this paper, we propose a bisection searching algorithm for the time-optimal profile to search the minimal transportation time (shown in Algorithm 3). First, search a feasible solution satisfying constraints $\mathcal{C}(N)$. Note that the interval of searching is $[0, N]$. Second, check whether a feasible solution can be found at the midpoint of interval and update the interval as shown in Algorithm 3. Third, repeat the second step iteratively until the tolerance error is satisfied.

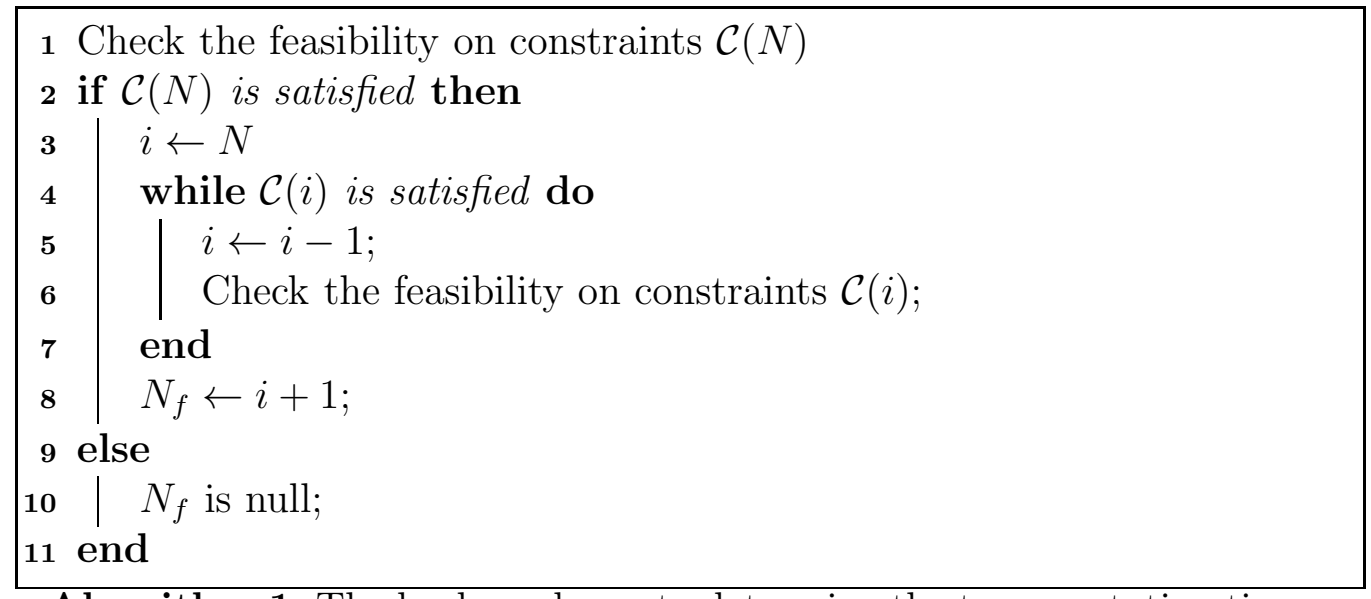

Algorithm 1: The backward way to determine the transportation time 


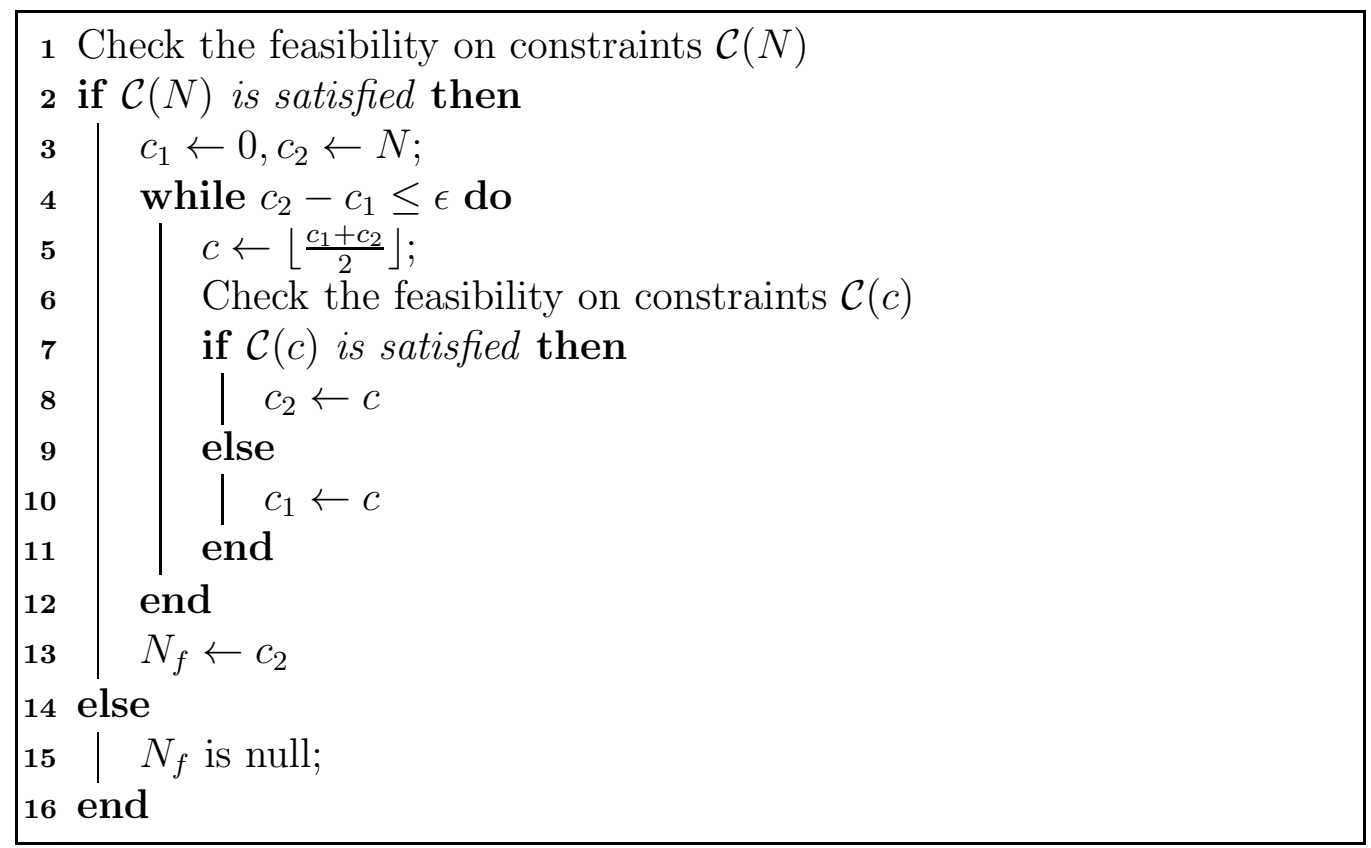

Algorithm 2: The bisection way to determine the transportation time

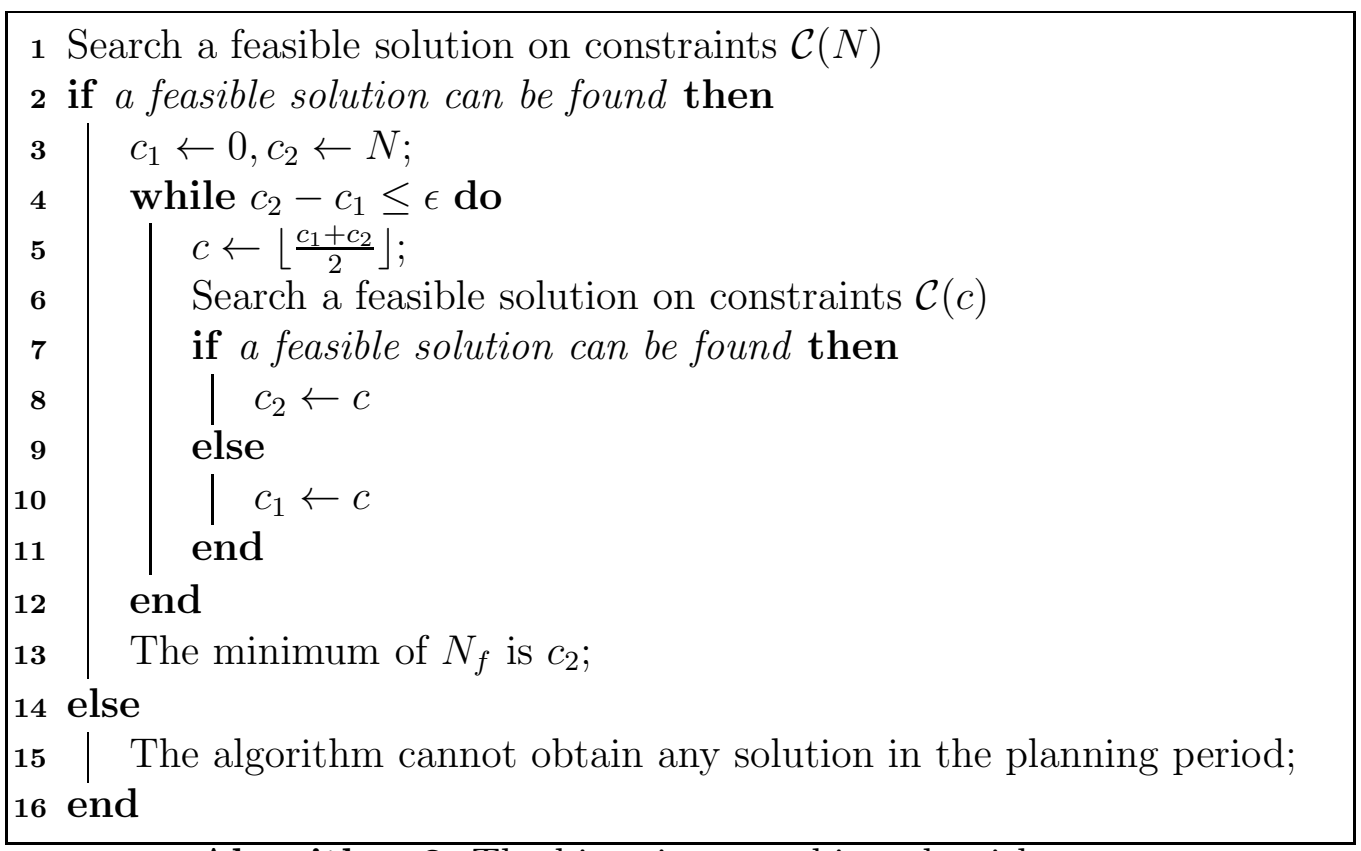

Algorithm 3: The bisection searching algorithm 


\section{Numerical Simulation}

The overhead crane system described in [9] is used to test our proposed models and the reference trajectories 1, 2, 3 and 4 . The physical parameters of the system are listed as follows

$$
m=1.025 \mathrm{~kg}, M=7 \mathrm{~kg}, l=0.75 \mathrm{~m}, g=9.8 \mathrm{~m} / \mathrm{s}^{2} .
$$

The desired trolley location in simulation is set as $p_{d}=0.6 \mathrm{~m}$, and the practical constraints are given as

$$
v_{m}=0.4 \mathrm{~m} / \mathrm{s}, a_{m}=0.2 \mathrm{~m} / \mathrm{s}^{2}, \theta_{m}=2^{\circ}, j_{m}=5 \mathrm{~m} / \mathrm{s}^{3} .
$$

The parameters for the friction model Eq. 4 are referred from the offline regression results in [28] as

$$
f_{r 0}=4.4, k_{r}=-0.5, \xi=0.01 \text {. }
$$

The simulation is conducted on a PC computer with Intel Core i7 CPU (3.4 $\mathrm{GHz}$ ) and 8 GB RAM. On the 64-bit Win-7 system, Matlab 2013 is installed as the software platform of coding and simulation.

\section{Test 1: comparisons of swing}

The evaluated planning period $T$ is $6 \mathrm{~s}$, and the sampling period $t_{0}$ is $0.01 \mathrm{~s}$. The duration of residual swing is defined as the last 2s, i.e., $N_{r}=200$. The optimization algorithm for solving the swing-optimal model is chosen as the fmincon function in Matlab toolbox. Note that fmincon is a simple example of solver in our simulations, other more complicated solvers may also be employed instead of it here. In the fmincon function, the algorithm type is set as "interior-point" and the maximum function evaluation times are $50 \cdot N$.

For each compared trajectory, the results of maximum swing angle, residual swing and their average are listed in Table 1. The swing-optimal trajectory has the smallest maximal swing angle $0.5278^{\circ}$. Although the residual swing of the optimal trajectory is larger than Trajectory 2, 3 and 4, but the average value of the maximal swing and the residue swing is the smallest among the compared trajectories. In Figure 3, the profiles of acceleration and swing angle are given. Due to the jerk constraint, the optimal trajectory has a smooth acceleration profile. In Figure 3, the same conclusion can be drawn namely that the maximum and average swing angles are smallest and the residual swing is close to zero. The profiles of acceleration and swing angle when $T=12 \mathrm{~s}$ are also given. The conclusion is clearer that due to longer planning period the maximum swing angle is much smaller than Trajectory 2, 3 and 4, and that the residual swing is close to zero. 
Table 1: Comparisons of swing

\begin{tabular}{|c|c|c|c|c|c|}
\hline Trajectory & Swing-optimal & 1 & 2 & 3 & 4 \\
\hline Maximal swing $\left(^{\circ}\right)$ & 0.5278 & 1.912 & 2.000 & 2.001 & 2.001 \\
\hline Residual swing $\left(^{\circ}\right)$ & 0.3600 & 0.8634 & $7.248 \times 10^{-3}$ & $6.622 \times 10^{-3}$ & $6.853 \times 10^{-3}$ \\
\hline Average $\left(^{\circ}\right)$ & $\mathbf{0 . 4 4 3 9}$ & 1.388 & 1.003 & 1.004 & 1.004 \\
\hline
\end{tabular}
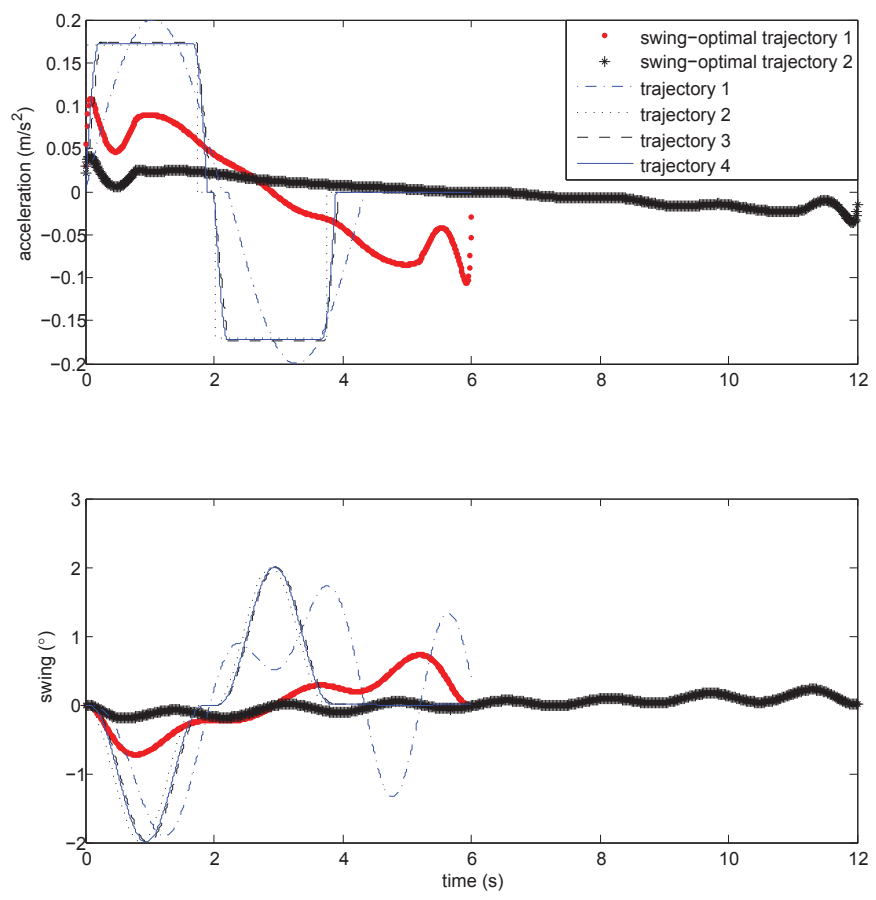

Figure 3: The trolley acceleration profile and the payload swing angle for the swing-optimal model: swing-optimal trajectory 1 with $T=6$ s; swingoptimal trajectory 2 with $T=12$ s. 

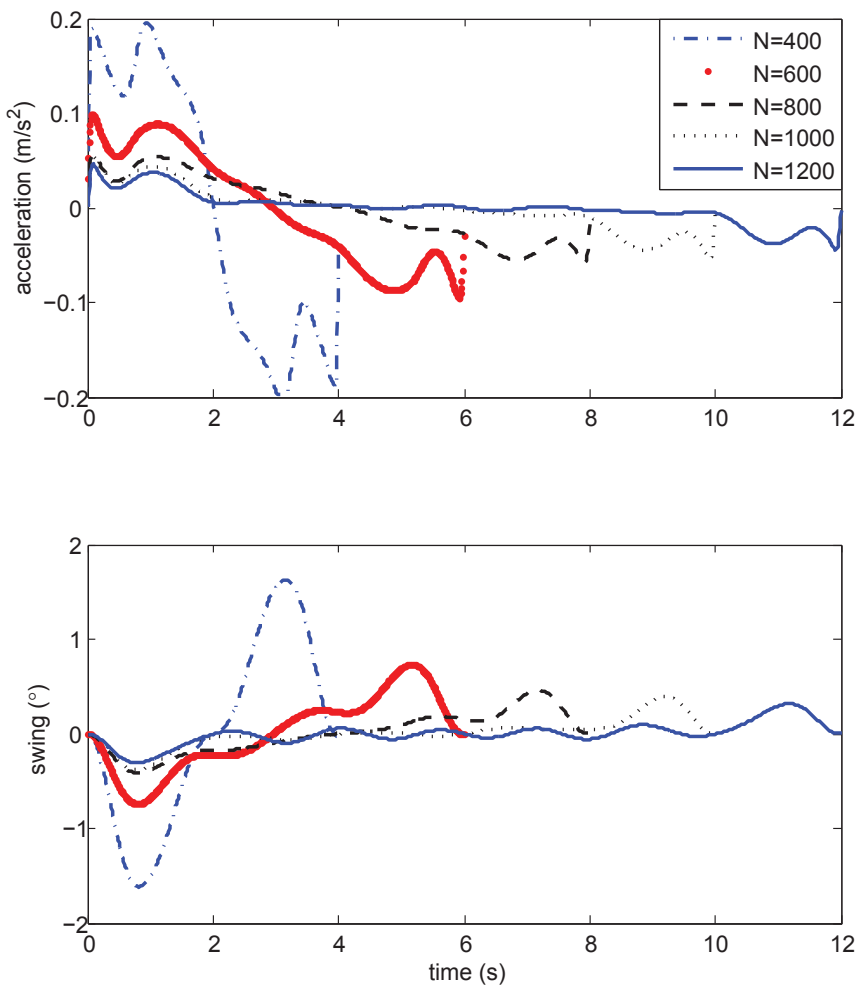

Figure 4: Profiles with different planning periods for the swing-optimal model 
In general, a long duration of transportation, i.e., a low average velocity, may reduce the swing of the payload. This can be verified by the following experiment to evaluate different planning periods, i.e. $4 \mathrm{~s}, 6 \mathrm{~s}, 8 \mathrm{~s}, 10 \mathrm{~s}$ and $12 \mathrm{~s}$. The resulting profiles of acceleration and swing angle are given in Figure 4. The maximal swing angle of $4 \mathrm{~s}$ period is the largest at $1.623^{\circ}$, and the maximum swing of $12 \mathrm{~s}$ period is the smallest at $0.319^{\circ}$. It can be concluded that the maximal swing is closely related to the evaluated planning period. The maximal swing decreases when the transportation period increases, but the change of maximal swing is small when planning period is larger than $10 \mathrm{~s}$.

\section{Test 2: comparisons of energy efficiency}

The evaluated planning period $T$ is $6 \mathrm{~s}$, and the sampling period $t_{0}$ is $0.01 \mathrm{~s}$. The optimization algorithm for solving the energy-optimal control model is chosen as the fmincon function in the Matlab toolbox. In the fmincon function, the algorithm type is set as "interior-point" and the maximum function evaluation times are $50 \cdot N$.

In Table 2, energy consumption and peak power during the entire transportation are listed for each trajectory. For the energy-optimal model, the lowest energy consumption and peak load (as bolded in the table) can be obtained. But the energy saving in this simulation is not large. One reason found is that the parameters of system referred from [9] are relatively small. When these parameters, such as maximum velocity, acceleration and rope length, turn larger, energy saving becomes more promising. For this energy-optimal trajectory, the maximum swing angle is $0.8948^{\circ}$ with the residue swing $0.2994^{\circ}$, and the average is $0.5971^{\circ}$. Profiles of acceleration, swing angle and power when $T=6$ and $12 \mathrm{~s}$ are given as in Figure 5. Compared with swing angles of Trajectory 1-4, energy-optimal trajectories still have smaller maximum and average swing angle as shown in the figure. As shown in the figure, the maximum swing angle of $T=12 \mathrm{~s}$ turns to be smaller than $T=6 \mathrm{~s}$ due to smoother acceleration. It can also be noticed that the optimal trajectory has a smooth power consumption while the other compared trajectories are not smooth. Although the optimal trajectory has a longer transportation time, the sacrifice of time efficiency is paid back with the improvement of energy efficiency.

Table 2: Comparisons of energy efficiency

\begin{tabular}{|c|c|c|c|c|c|}
\hline Trajectory & Energy-optimal & 1 & 2 & 3 & 4 \\
\hline Energy usage $(\mathrm{J})$ & $\mathbf{2 . 6 2 6}$ & 2.651 & 2.653 & 2.652 & 2.653 \\
\hline Peak power $(\mathrm{W})$ & $\mathbf{0 . 7 0 2}$ & 1.323 & 1.647 & 1.602 & 1.589 \\
\hline
\end{tabular}

The relation of energy efficiency and time efficiency has not been clearly stated in the literature. In this section, an experiment with different planning periods 

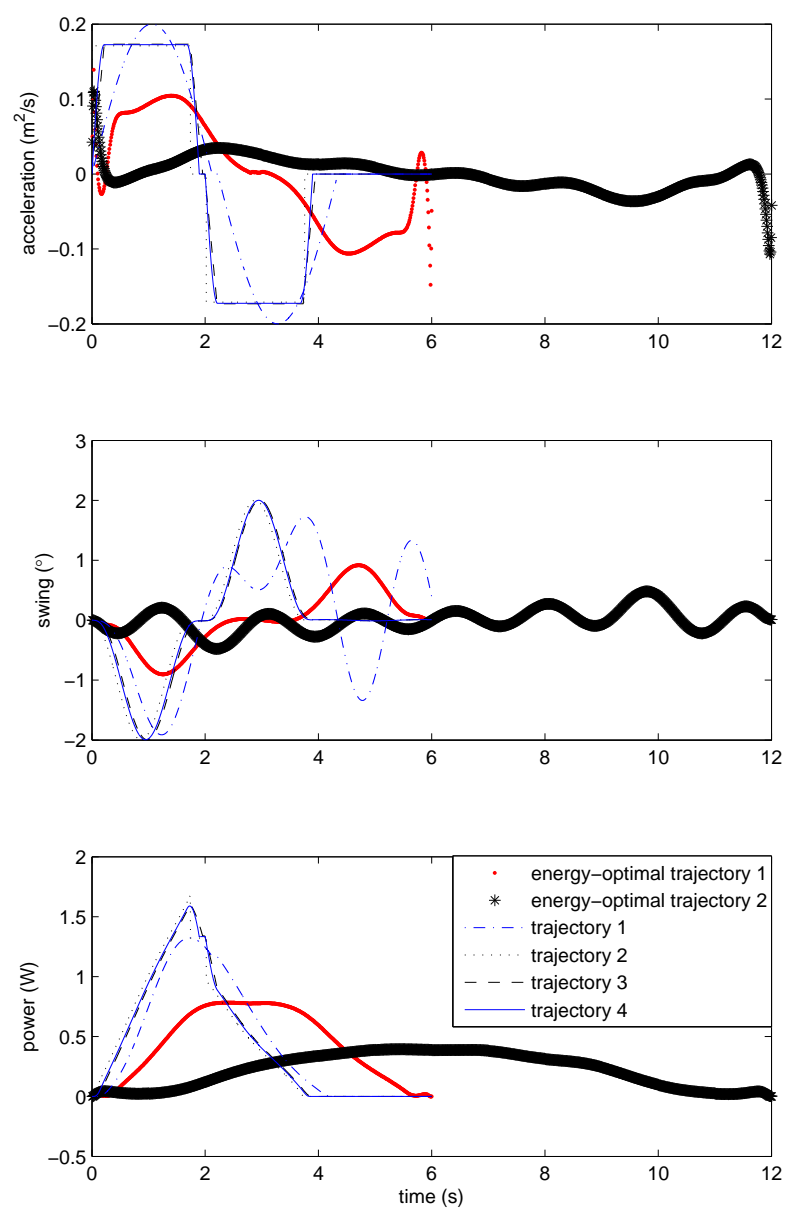

Figure 5: Profiles of acceleration, swing angle and power for the energyoptimal model: energy-optimal trajectory 1 with $T=6 \mathrm{~s}$; energy-optimal trajectory 2 with $T=12 \mathrm{~s}$ 
has been done to evaluate such relation. Planning periods are set to be $4 \mathrm{~s}, 6 \mathrm{~s}$, $8 \mathrm{~s}, 10 \mathrm{~s}$, and $12 \mathrm{~s}$, respectively. Then after solving the energy-optimal model over each period, energy consumptions can be obtained. When the period is $4 \mathrm{~s}$, energy consumption is the largest at $2.6534 \mathrm{~J}$. When the period is $12 \mathrm{~s}$, energy consumption is the smallest at $2.5777 \mathrm{~J}$. When the planning period gets larger than $10 \mathrm{~s}$, the change of peak power is close to zero and the energy consumption has slow or no decrease as shown in Figure 6.
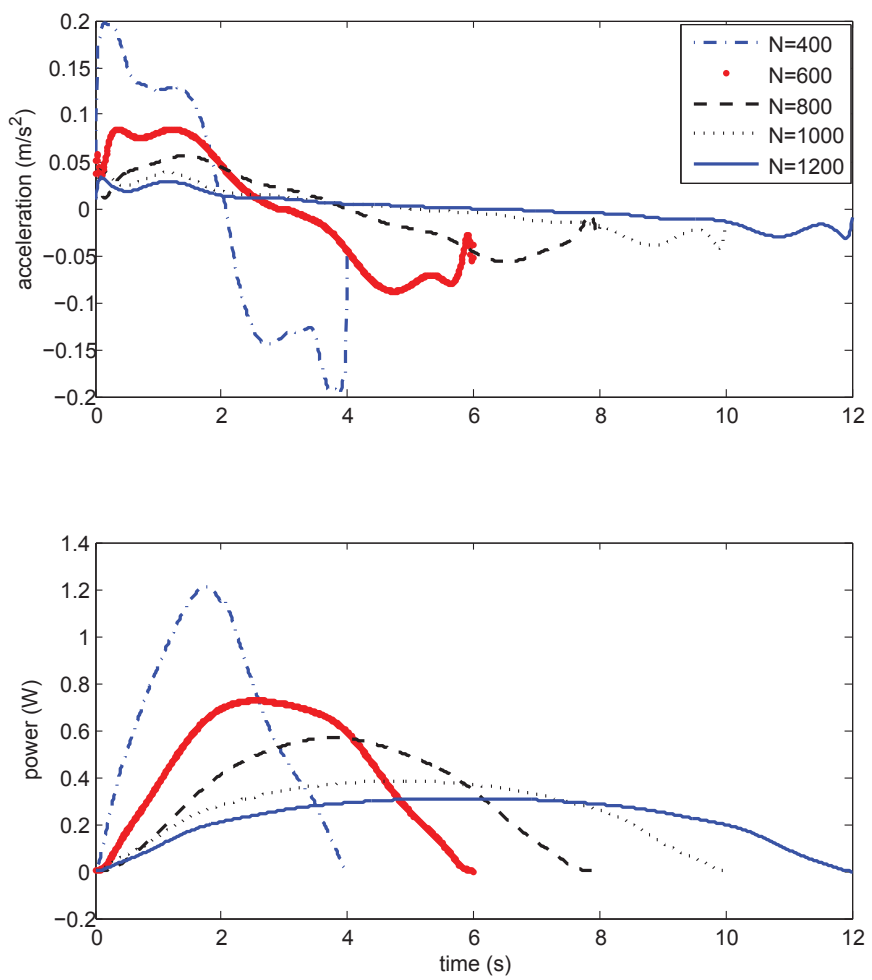

Figure 6: Profiles with different planning periods for the energy-optimal model

\section{Test 3: comparisons of time efficiency}

The evaluated transportation period $T$ is $6 \mathrm{~s}$, and the sampling period $t_{0}$ is $0.01 \mathrm{~s}$. The optimization algorithm for solving the time-optimal control model is chosen as the fmincon function in the Matlab toolbox. In the fmincon function, the maximum function evaluation times are $50 \cdot N$. The algorithm type is set as "active-set", because "active-set" is suitable to find a feasible solution for the time optimal model. The number of iterations in the bisection algorithm is set to 10 . 
As mentioned in Section 3, Trajectory 2 has the shortest transportation time among the five trajectories reviewed, but the jerk at certain time is infinite. For the tested crane system, the transportation time of Trajectory 2 is $3.66 \mathrm{~s}$. When ignoring the jerk constraint in our time optimal control model, we can obtain an optimal trajectory with transportation time of $3.62 \mathrm{~s}$. The profile of acceleration is given in Figure 7.

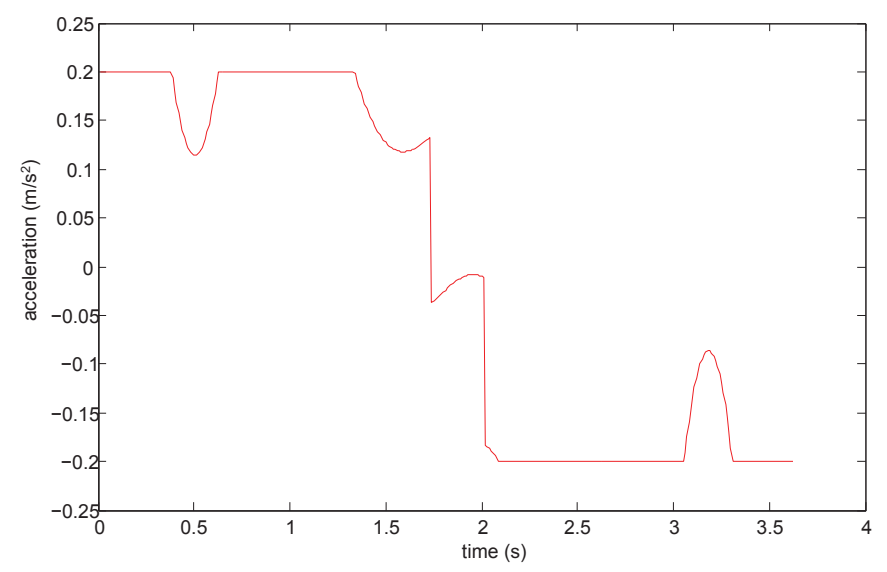

Figure 7: Profiles of acceleration without the jerk constraint for the time optimal model

The optimal trajectory with the jerk constraint $\left(j_{m}=5\right)$ is given in Figure 8 . The optimal time of transportation is $3.65 \mathrm{~s}$, while the transportation time of Trajectory 1,3 and 4 is $4.08 \mathrm{~s}, 3.76 \mathrm{~s}$ and $3.73 \mathrm{~s}$, respectively. Figure 8 shows profiles of displacement, acceleration and jerk. The crane using the optimal trajectory can arriver at the destination earlier than using other trajectories. It can be noticed that the jerk of the optimal trajectory never violates the permitted maximal jerk, which can be set by users according to different requirements. For example, when the actuating motor of the crane system is retrofitted, the maximum of jerk can be changed according to the performance of the new motor.

\section{Conclusion}

In view of safety, energy efficiency and time efficiency, three optimal motion planning strategies for overhead cranes have been proposed to provide three trajectories with the lowest swing, energy consumption, and transportation time respectively. These optimal planning strategies have considered practical and physical constraints such as maximal swing and jerk. Note that these trajectories obtained 

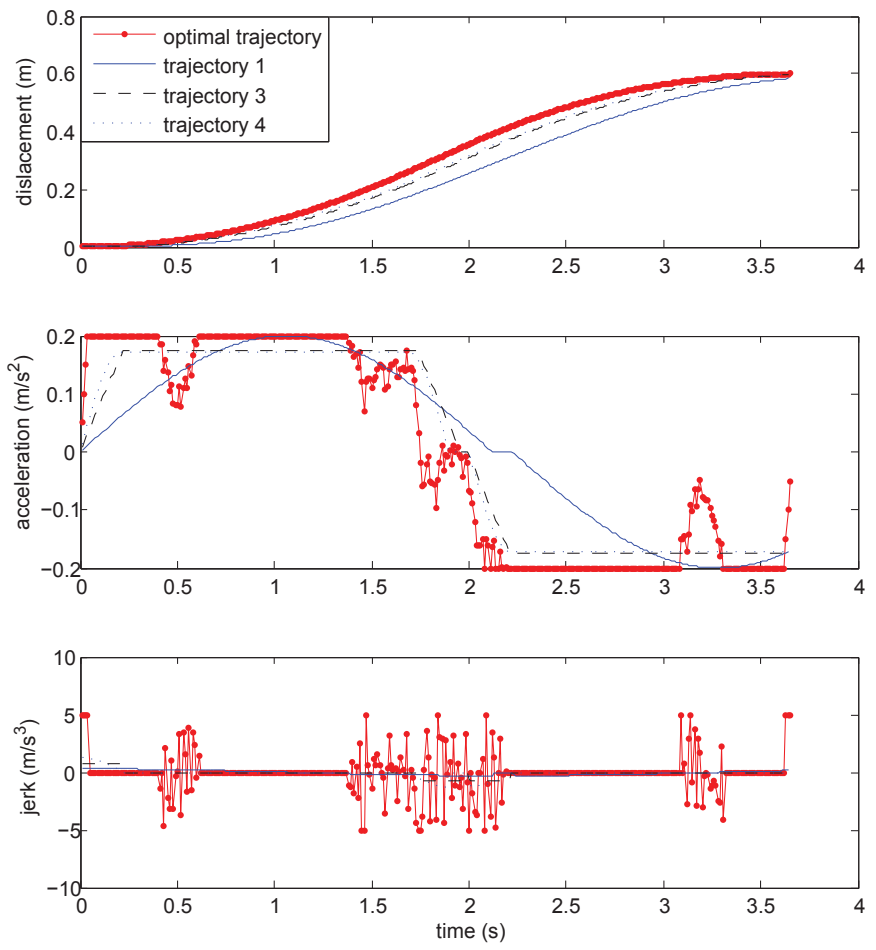

Figure 8: Profiles of acceleration with jerk constraints for time optimal model 
by off-line computation can be utilized as inputs of open loop control schemes, or as tracking references of closed-loop control schemes.

In this paper, energy efficiency of crane is newly modeled under practical and physical constraints, including maximal swing and jerk. The result of the energyoptimal model can reduce energy consumption and peak power, which may be useful in the incentive electricity market. The time and swing-optimal strategies can also achieve better performance than existing strategies in terms of time efficiency and safety. In practise, decision makers can choose one optimal strategy among these three proposed ones depending on the requirements of work. As these three objectives are competing, we have only considered each of them separately in this paper. Methods of integrating three objectives can be reasonably extended, but that is beyond the scope of this paper. In future work, the phases of hoisting and lowering will be combined with the horizontal transportation for complete motion planning using the proposed methods, and the closed-loop methods may be proposed for tracking the planned trajectory and updating the trajectory online. The proposed strategies of motion planning are developed on two dimensional crane systems, and they are possibly extended on three or more dimensional systems as part of future work.

\section{References}

[1] K. Peng, W. Singhose, and S. Gurleyuk, "Initial investigations of hand-motion crane control with double-pendulum payloads," in Proceedings of the 2012 American Control Conference, 2012, pp. 6270-6275, 2012.

[2] Q. H. Ngo and K.-S. Hong, "Sliding-mode antisway control of an offshore container crane," IEEE/ASME Transactions on Mechatronics, vol. 17, no. 2, pp. 201-209, 2012.

[3] Z. N. Masoud, "Effect of hoisting cable elasticity on anti-sway controllers of quay-side container cranes," Nonlinear Dynamics, vol. 58, no. 1-2, pp. 129140, 2009.

[4] W. Blajer and K. Kotodziejczyk, "Motion planning and control of gantry cranes in cluttered work environment," IET Control Theory Applications, vol. 1, pp. 1370-1379, Sept 2007.

[5] T. Heyden and C. Woernle, "Dynamics and flatness-based control of a kinematically undetermined cable suspension manipulator," Multibody System Dynamics, vol. 16, no. 2, pp. 155-177, 2006. 
[6] O. Sawodny, H. Aschemann, and S. Lahres, "An automated gantry crane as a large workspace robot," Control Engineering Practice, vol. 10, no. 12, pp. $1323-1338,2002$.

[7] W. Blajer and K. Kolodziejczyk, "Improved DAE formulation for inverse dynamics simulation of cranes," Multibody System Dynamics, vol. 25, no. 2, pp. 131-143, 2011.

[8] H.-H. Lee, "Motion planning for three-dimensional overhead cranes with high-speed load hoisting," International Journal of Control, vol. 78, no. 15, pp. 875-886, 2005.

[9] N. Sun, Y. Fang, X. Zhang, and Y. Yuan, "Transportation task-oriented trajectory planning for underactuated overhead cranes using geometric analysis," IET Control Theory Applications, vol. 6, no. 10, pp. 1410-1423, 2012.

[10] C.-Y. Chang and H. Wijaya Lie, "Real-time visual tracking and measurement to control fast dynamics of overhead cranes," IEEE Transactions on Industrial Electronics, vol. 59, no. 3, pp. 1640-1649, 2012.

[11] N. A. Nayfeh and W. T. Baumann, "Nonlinear analysis of time-delay position feedback control of container cranes," Nonlinear Dynamics, vol. 53, no. 1-2, pp. 75-88, 2008.

[12] M.-S. Park, D. Chwa, and S.-K. Hong, "Antisway tracking control of overhead cranes with system uncertainty and actuator nonlinearity using an adaptive fuzzy sliding-mode control," IEEE Transactions on Industrial Electronics, vol. 55, no. 11, pp. 3972-3984, 2008.

[13] N. Sun and Y. Fang, "New energy analytical results for the regulation of underactuated overhead cranes: An end-effector motion-based approach," IEEE Transactions on Industrial Electronics, vol. 59, no. 12, pp. 4723-4734, 2012.

[14] K. Hekman and W. Singhose, "Feedback control for suppression of crane payload oscillation using on-off commands," in Proceedings of the 2006 American Control Conference, 2006, pp. 1784-1789, 2006.

[15] K. L. Sorensen, W. Singhose, and S. Dickerson, "A controller enabling precise positioning and sway reduction in bridge and gantry cranes," Control Engineering Practice, vol. 15, no. 7, pp. 825 - 837, 2007.

[16] Z. N. Masoud and A. H. Nayfeh, "Sway reduction on container cranes using delayed feedback controller," Nonlinear Dynamics, vol. 34, no. 3-4, pp. 347358, 2003. 
[17] W. Singhose, L. Porter, M. Kenison, and E. Kriikku, "Effects of hoisting on the input shaping control of gantry cranes," Control Engineering Practice, vol. 8, no. 10, pp. 1159 - 1165, 2000.

[18] S. Garrido, M. Abderrahim, A. Gimenez, R. Diez, and C. Balaguer, "Antiswinging input shaping control of an automatic construction crane," IEEE Transactions on Automation Science and Engineering, vol. 5, no. 3, pp. 549$557,2008$.

[19] K.-T. Hong, C.-D. Huh, and K.-S. Hong, "Command shaping control for limiting the transient sway angle of crane systems," International Journal of Control, Automation, and Systems, vol. 1, no. 1, pp. 43-53, 2003.

[20] M. Moon, H. Vanlandingham, and Y. Beliveau, "Fuzzy time optimal control of crane load," in Proceedings of the 35th IEEE Conference on Decision and Control, 1996, vol. 2, pp. 1127-1132 vol.2, 1996.

[21] A. Piazzi and A. Visioli, "Optimal dynamic-inversion-based control of an overhead crane," IEE Proceedings of Control Theory and Applications, vol. 149, no. 5, pp. 405-411, 2002.

[22] K. Terashima, Y. Shen, and K. Yano, "Modeling and optimal control of a rotary crane using the straight transfer transformation method," Control Engineering Practice, vol. 15, no. 9, pp. 1179 - 1192, 2007.

[23] W. Blajer and K. Kolodziejczyk, "Control of underactuated mechanical systems with servo-constraints," Nonlinear Dynamics, vol. 50, no. 4, pp. 781-791, 2007.

[24] N. Sun and Y. Fang, "An efficient online trajectory generating method for underactuated crane systems," International Journal of Robust and Nonlinear Control.

[25] Y. Fang, B. Ma, P. Wang, and X. Zhang, "A motion planning-based adaptive control method for an underactuated crane system," IEEE Transactions on Control Systems Technology, vol. 20, no. 1, pp. 241-248, 2012.

[26] S. Zhang and X. Xia, "Modeling and energy efficiency optimization of belt conveyors," Applied Energy, vol. 88, no. 9, pp. 3061 - 3071, 2011.

[27] C. Makkar, G. Hu, W. Sawyer, and W. Dixon, "Lyapunov-based tracking control in the presence of uncertain nonlinear parameterizable friction," IEEE Transactions on Automatic Control, vol. 52, no. 10, pp. 1988-1994, 2007. 
[28] N. Sun, Y. Fang, Y. Zhang, and B. Ma, "A novel kinematic coupling-based trajectory planning method for overhead cranes," IEEE/ASME Transactions on Mechatronics, vol. 17, no. 1, pp. 166-173, 2012.

[29] E. Abdel-Rahman, A. Nayfeh, and Z. Masoud, "Dynamics and control of cranes: A review," Journal of Vibration and Control, vol. 9, no. 7, pp. 863908, 2003.

[30] G. Starr, "Swing-free transport of suspended objects with a path-controlled robot manipulator," Journal of dynamic systems, measurement, and control, vol. 107, no. 1, pp. 97-100, 1985. 